
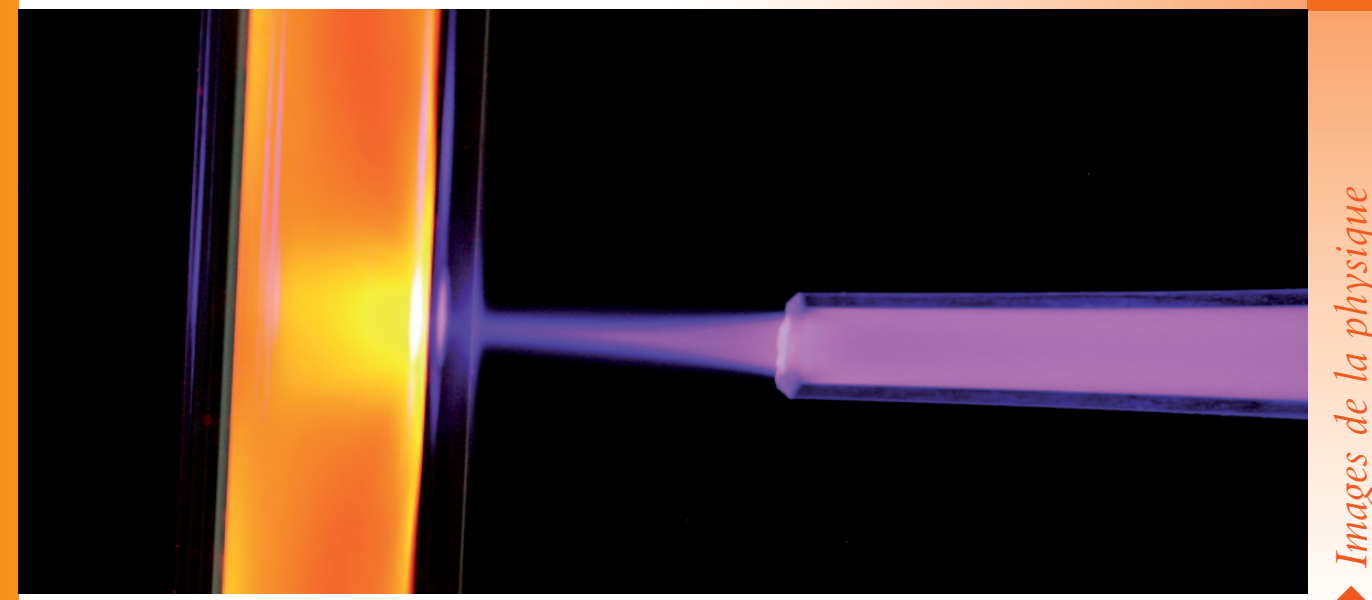

Transfert d'une colonne de plasma d'un tube issu d'un réacteur DBD annulaire parcouru par de l'hélium, dans un tube sans électrode parcouru par du néon (voir encadré 1, p. 19).

\title{
Applications thérapeutiques des plasmas froids atmosphériques
}

Jean-Michel Pouvesle (jean-michel.pouvesle@univ-orléans.fr) et Éric Robert

Groupe de Recherches sur l'Énergétique des Milieux Ionisés (GREMI), UMR 7344 CNRS-Université d'0rléans, BP 6744, 45067 Orléans Cedex 2

\section{Depuis quelques années,}

des avancées importantes sont réalisées sur les applications

des plasmas froids à la biologie. À côté des secteurs déjà bien identifiés que sont la stérilisation/ décontamination, l'ingénierie de surface, les biomatériaux et l'ingénierie des tissus, les applications thérapeutiques des plasmas connaissent un développement extraordinaire. Si les processus impliqués sont loin d'être bien compris, les premières applications cliniques font leur apparition.

Après avoir évoqué

les sources et les réacteurs adaptés, nous présenterons quelques développements récents importants relatifs à l'effet antitumoral des plasmas froids.
Dans un proche avenir, les médecins parlant de "plasma " dans le cadre de leurs activités devront sans doute préciser de quel plasma il s'agit : plasma sanguin ou gaz ionisé. En effet, les plasmas froids hors équilibre à la pression atmosphérique (PFA), créés par décharge électrique, présentent des caractéristiques qui les rendent très attractifs pour le développement de nouveaux procédés dans le domaine médical et particulièrement pour de nouvelles applications thérapeutiques. Ce domaine, maintenant dénommé "médecine plasma ", a connu des développements spectaculaires au cours des dernières années, surtout depuis 2005. Il a notamment été montré que de très nombreuses applications pouvaient être abordées, concernant aussi bien la coagulation du sang, la prévention du développement de bactéries sur des organes opérés, que le secteur dentaire, la dermatologie, les soins sur des plaies persistantes ou des ulcères, la médecine régénérative ou le traitement de tumeurs, pour ne citer que quelques exemples.

L'engouement suscité par les recherches concernant les applications biomédicales des plasmas froids est tel que des sessions spécialisées sont organisées dans tous les grands symposiums ou conférences plasmas. Le domaine où les progrès ont été les plus spectaculaires est certainement celui du traitement des blessures chroniques (plaies ou ulcères), pour lequel des essais cliniques sur un grand nombre de patients ont déjà été réalisés. En France, les travaux effectués à Orléans par le GREMI et le CIPATAAM, en collaboration avec le $\mathrm{CBM}^{(2)}$, ont permis d'obtenir des résultats majeurs concernant l'effet antitumoral des plasmas et ainsi d'occuper une position de leader dans ce domaine.

\section{Pourquoi les PFA, pourquoi maintenant?}

Les plasmas sont des sources naturelles d'espèces actives. Dans l'air, ils peuvent facilement conduire à la production d'espèces réactives de l'oxygène et de l'azote ${ }^{(b)}$, dont le rôle a déjà été mis en évidence dans des thérapies établies. Que le plasma soit produit directement dans l'air ou dans un gaz rare débouchant à l'air libre, il conduit à des transferts d'énergie sur les molécules d'azote et d'oxygène et produit ainsi des radicaux $\mathrm{OH}$ ou NO. Ces radicaux sont connus, pour le premier, comme l'un des précurseurs de la chaîne de processus renforçant le stress oxydatif ${ }^{(c)}$ au sein des cellules et tissus vivants, ou favorisant l'angiogenèse $^{(\mathrm{d})}$ ou la réparation tissulaire pour le second. On peut d'emblée souligner que les plasmas permettront un traitement localisé et non pas systémique comme c'est le cas lors de l'absorption de médicaments, et qu'ils ne conduiront pas en général à la production de produits ou sous-produits à éliminer par le corps ou à retraiter. 
$>>$

Les deux principaux paramètres qui contrôlent les plasmas doivent s'accommoder de la nature vivante de la cible : pression atmosphérique et température d'utilisation inférieure à $42^{\circ} \mathrm{C}$ (température ambiante, si possible). Notons, cependant, que des plasmas thermiques, beaucoup plus chauds (quelques centaines à quelques milliers de degrés), sont déjà présents dans les salles d'opération. Il s'agit de « couteaux à argon " ou d'électrocoagulateurs. Ces minitorches plasma radiofréquence (RF), fonctionnant en général avec de l'argon ou des mélanges argon-hélium à des débits de quelques litres par minute, sont utilisées en chirurgie pour leurs caractéristiques thermiques. Elles permettent une coagulation rapide lors de la découpe de tissus ou d'organes, évitant ainsi des saignements importants.

Les plasmas froids, le plus souvent à basse pression, sont depuis plus de vingt-cinq ans utilisés dans le cadre de la stérilisation et de la décontamination d'objets fragiles. Ils ont démontré depuis longtemps un pouvoir bactéricide et antimicrobien d'un grand intérêt pour le secteur de la santé. Mais jusque récemment, les préoccupations des spécialistes des plasmas froids à la pression atmosphérique, éloignées du monde de la biologie, étaient surtout focalisées sur la production de sources de rayonnement et sur la transformation des effluents gazeux à des fins de dépollution. La réalisation de systèmes compacts, permise par les progrès récents de l'électronique - plus particulièrement de l'électronique de mise en forme et de commande des impulsions haute tension -, conjuguée à l'engouement pour les recherches pluridisciplinaires, ont créé les conditions favorables au rapprochement avec la communauté des biologistes. Il existe maintenant à travers le monde des laboratoires et des instituts ${ }^{(e)}$ complètement dédiés à cette thématique, où sont rassemblés des spécialistes de l'ensemble des disciplines impliquées. En France, les principaux laboratoires travaillant dans ce domaine sont réunis depuis janvier 2010 au sein du GDR 3374 ABioPlas : Applications Biomédicales des Plasmas.

\section{Des réacteurs adaptés}

Les réacteurs permettant de produire les plasmas adéquats (fig. 1) sont essentiellement de deux types.

1) Les systèmes de décharges à barrières diélectriques (DBD, fig. 1a), où l'une des électrodes est recouverte d'une couche diélectrique évitant toute production d'arc. Ces décharges, à la pression atmosphérique, conduisent en général à la production d'un grand nombre de petits filaments de plasma répartis aléatoirement à la surface de l'électrode. Le milieu plasmagène (mélange de gaz où se développe le plasma) est directement l'air ambiant. Le réacteur doit être proche (quelques $\mathrm{mm}$ ) de la surface à traiter, et la cible est la deuxième électrode. On utilise généralement la dénomination de DBD à électrode flottante, ou FE DBD (Floating Electrode). Ces dispositifs sont alimentés, la plupart du temps, en haute tension alternative (quelques $\mathrm{kV}$ ) à haute fréquence (de quelques $\mathrm{kHz}$ à quelques dizaines de $\mathrm{kHz}$ ). Ce type de réacteur est particulièrement adapté aux traitements de surface et ne génère pas d'ultraviolets C (de longueurs d'ondes comprises entre 200 et $280 \mathrm{~nm}$ ).

2) Les jets de plasmas eux-mêmes, divisés en deux grandes catégories : les plasmas radiofréquence et micro-onde (fig. 1b) et les plasmas créés par DBD annulaire (fig. 1c). Initiés dans des gaz rares, essentiellement hélium et argon, ils débouchent dans l'air sous forme d'une plume plasma dont les espèces actives et les photons transferent leur énergie sur les composants de l'air en périphérie. Le débit de gaz (1 à 10 litres par minute) a une forte influence sur la longueur de la plume et la production des espèces actives. Dans le cas des plasmas radiofréquence, dont la température en sortie immédiate du réacteur $\left(70-80^{\circ} \mathrm{C}\right)$ est significativement supérieure à la température de traitement requise, le réacteur doit être maintenu à une distance suffisante de la cible, de l'ordre du $\mathrm{cm}$. Les jets de plasma offrent une grande souplesse d'utilisation (adaptation des paramètres de débit de gaz, mélange de gaz, tension, fréquence, cycle de décharge), particulièrement adaptée à des traitements précis ou robotisés.

Il est important de noter que, quel que soit le type de décharge utilisé parmi celles qui sont mentionnées ci-dessus, le réacteur

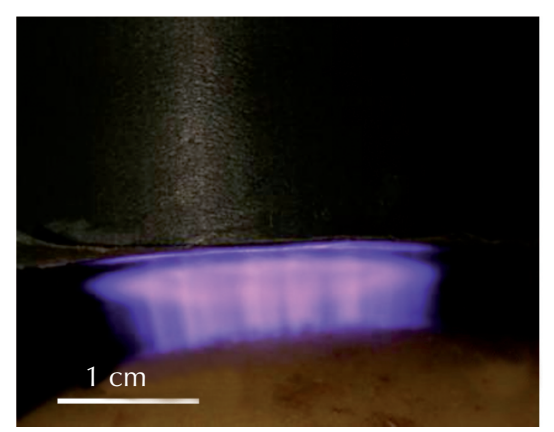

a

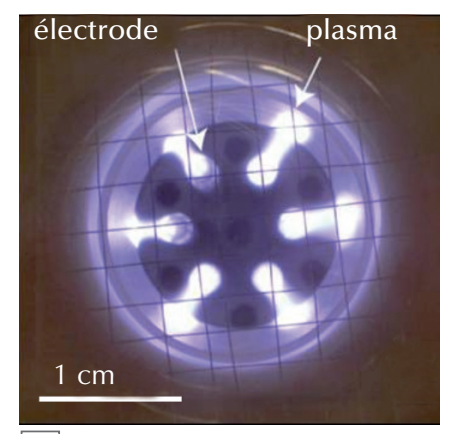

$\mathrm{b}$

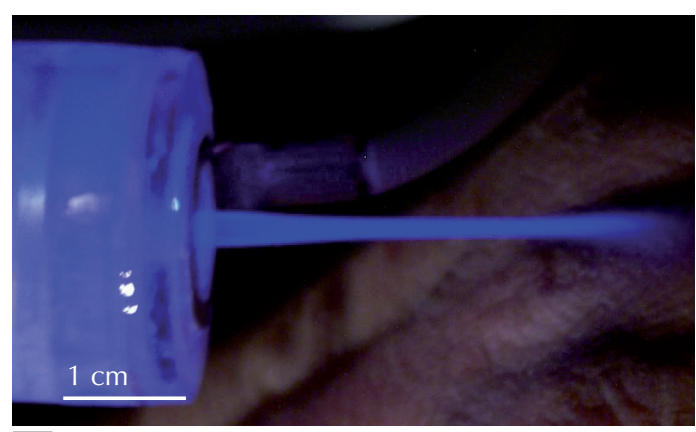

C

1. Sources de plasma à la pression atmosphérique pour des applications thérapeutiques.

(a) Système de décharges à barrières diélectriques à électrode flottante (FE DBD) dans l'air, avec gap de 4 mm (sous 20 kV à 1 kHz) (GREMI Orléans, France). La surface à traiter est en bas de la photo.

(b) Torche plasma radiofréquence MicroPlaSter à flux d'argon de 2,2 litres par minute (100 W à 2,3 GHz) (Adtec, UK, et MPE, Garching, Allemagne). Six petits plasmas sont créés entre six électrodes en acier inoxydable et la surface intérieure d'un cylindre d'aluminium. Les jets de plasma sortent perpendiculairement au plan de la figure. (c) Jet de plasma produit par un système de décharges à barrières diélectriques annulaires à flux d’hélium de 3,5 litres par minute (sous 5 kV à 5 kHz) (LPEI, Norfolk, USA). 
plasma se trouve toujours à proximité de la cible (tissu, organe), ce qui peut poser des problèmes de manipulation ou d'accès. Pour pallier ce problème, le GREMI a développé une nouvelle catégorie de réacteurs, appelés Plasma Gun (voir encadré 1), qui permettent de générer des plumes plasma à très longue distance (de quelques dizaines de $\mathrm{cm}$ à plus d'un mètre cinquante selon les paramètres de décharge) dans des tubes ou des capillaires diélectriques, dont le diamètre varie de quelques dizaines de micromètres à un centimètre. Ces réacteurs sont particulièrement adaptés aux traitements de cibles difficiles d'accès ou nécessitant une voie endoscopique.

\section{Des essais cliniques}

Parmi tous les résultats obtenus récemment à travers le monde en médecine plasma, on se contentera ici d'évoquer tout d'abord une application phare, puis de détailler les résultats obtenus à Orléans au sein du projet PLASMED.

Le traitement des plaies chroniques et des ulcères est l'application des plasmas la plus avancée, pour laquelle des essais cliniques ont été menés en Allemagne sur plusieurs centaines de patients présentant des ulcères infectés du bas de la jambe. Les résultats sont très encourageants (fig. 2) et démontrent l'intérêt des recherches dans ce domaine. Les traitements sont réalisés avec le dispositif appelé MicroPlaSter (Microwave Plasma Sterilizer, figs. $1 \mathrm{~b}$ et $2 \mathrm{a}$ ). Le plasma d'argon,

\〉

\section{Le Plasma Guin}
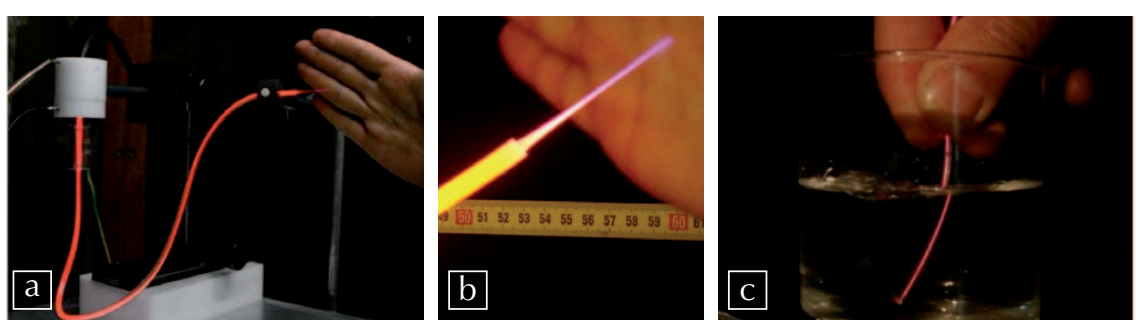

E1. (a) Plasma Gun (néon, $400 \mathrm{~cm}^{3} /$ minute, 1 kHz). (b) Plume Plasma Gun (néon, $1500 \mathrm{~cm}^{3} /$ minute, 10 Hz). (c) Plume Plasma Gun débouchant dans l'eau à la sortie d'un capillaire de 200 um de diamètre (néon, 50 cm³/minute, 50 Hz).

Les chercheurs du GREMI ont mis au point un nouveau type de générateur de plasmas froids à la pression atmosphérique (brevet CNRS/Université d'Orléans), qui permet de générer des colonnes pulsées de plasma à longue distance (de quelques $\mathrm{cm}$ à plus d'un mètre cinquante), dans des tubes ou capillaires diélectriques souples ou rigides (plastique, céramique, verre) (fig. E1a). À l'extrémité du capillaire, une plume plasma est produite dans I'air (fig. E1b) ou dans tout autre milieu, même liquide (fig. E1c). Ce dispositif permet d'amener le milieu réactif sur la zone de traitement et à distance du système de décharge à haute tension. Les colonnes de plasma se propagent dans les tubes à très grande vitesse, de quelques $10^{6}$ à quelques $10^{8} \mathrm{~cm} / \mathrm{s}$, selon les conditions de décharge et la distance au réacteur, et peuvent parcourir de quelques millimètres à quelques centimètres supplémentaires à l'air libre. Ce nouveau générateur de plasmas atmosphériques peut aussi bien fonctionner en régime monocoup qu'à des taux de répétition élevés de plusieurs $\mathrm{kHz}$, donnant l'illusion d'un jet de plasma continu (fig. E1).

Ce procédé permet de bien doser le nombre d'espèces actives générées près de la surface à traiter. Alors que les jets de plasma classiques nécessitent des débits de gaz rares élevés, de 1 à 10 litres/minute, le Plasma Gun peut fonctionner à des débits extrêmement faibles, jusqu'à quelques $\mathrm{cm}^{3} /$ minute, permettant des applications impossibles avec les autres systèmes, notamment à l'intérieur du corps. Les colonnes de plasma sont constituées d'une tête lumineuse, qui correspond à un front d'ionisation intense, et d'un canal beaucoup moins lumineux, qui relie électriquement ce front au réacteur. L'impédance du canal plasma créé va influer sur la tension transmise, et donc sur le champ électrique créé en avant de la colonne, et finalement sur le taux d'ionisation et la vitesse de propagation. Les colonnes de plasma peuvent se diviser lorsqu'elles débouchent sur des ramifications (voir la photographie en tête d'article, p. 17).

Au-delà des traitements endoscopiques, le Plasma Gun est bien adapté pour des traitements dans des cavités ou des structures complexes. Ses applications dépassent largement le domaine de la biologie et concernent, entre autres, les traitements de surfaces ou la commutation rapide.

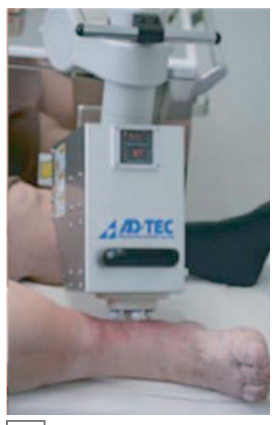

a

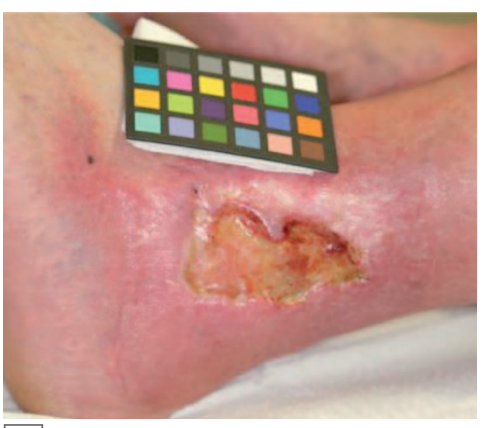

b 1 traitement

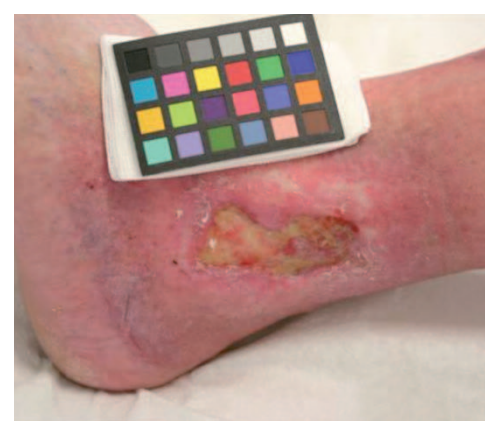

15 traitements

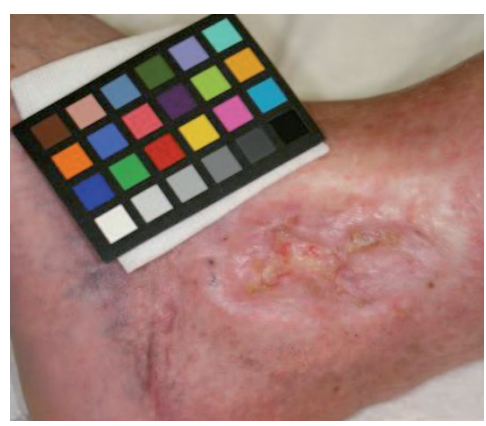

33 traitements

2. Traitement d'un ulcère par plasma d'argon.

(a) Dispositif de traitement MicroPlaSter, développé en partenariat entre le MPE(a) à Garching et l'entreprise Adtec au Royaume-Uni.

(b, c, d) Évolution de l'ulcère après 1, 15 et 33 traitements de deux minutes. La mire colorée permet de corriger les variations des conditions de photographie des plaies (éclairement, ombre...), la « couleur » de la plaie étant l'un des indicateurs de la cicatrisation. Données de l'hôpital Schwabing de Munich/MPE, Garching, Allemagne (G. Isbary et al.). 
appliqué (en plus des soins courants) durant deux minutes tous les jours jusqu'à guérison ou amélioration suffisante, permet à la fois une débactérisation de la plaie et une aide à la reconstruction des tissus. De manière à travailler dans des conditions parfaitement reproductibles, et dans la mesure où la température de sortie du plasma est légèrement trop élevée, la distance au tissu traité est contrôlée au moyen d'un dispositif à ultrasons. Il est important de noter que les traitements n'engendrent pas d'effets secondaires ni de douleurs particulières.

\section{Effet antitumoral des plasmas froids}

L'utilisation des PFA dans le traitement de certains types de cancers apparait aussi extrêmement prometteuse. Dès 2009 a eu lieu la première mise en évidence d'un effet antitumoral in vivo sur des souris porteuses de tumeurs cérébrales humaines ${ }^{(t)}$, avec des effets à court terme (régression de la masse tumorale) et des effets à long terme (augmentation de $60 \%$ de l'espérance de vie des souris). Il faut noter que ce type de tumeur est connu pour être particulièrement chimio- et radiorésistant. Ces travaux ont été les premiers d'une longue série d'expériences in vitro et in vivo, qui ont conduit très récemment à des résultats remarquables sur l'association plasma/chimiothérapie appliquée au cancer du pancréas.

Les premières expériences ont été réalisées avec un dispositif du type de la figure 1a, en traitant les tumeurs greffées sur le haut de cuisse à travers la peau de souris "nude"(g). Il a d'abord été montré que l'application du plasma sur des temps longs (supérieurs à dix minutes) n'entraînait pas d'effets secondaires à court terme - augmentation de la température de la peau (une augmentation de 2 à $3^{\circ} \mathrm{C}$ est néanmoins mesurée, mais disparaît immédiatement à l'arrêt du plasma), ou modification des rythmes cardiaque ou respiratoire des animaux - et n'engendrait pas non plus d'effets sur le comportement.

Les études in vitro ont été menées sur différentes lignées de cellules cancéreuses, cérébrales, du côlon, du pancréas et du poumon. Les expériences ont montré le rôle important joué par les espèces réactives de l'oxygène. Les plasmas induisent des cassures des doubles brins de l'ADN, conduisant à une apoptose (mort cellulaire programmée) massive des cellules, mise en évidence en particulier par bioluminescence (voir encadré 2). De plus, on a observé in vitro et in vivo un blocage du cycle cellulaire des cellules tumorales, conduisant à un arrêt de leur prolifération.
Les mécanismes mis en jeu sont proches de ceux qui sont induits par radiothérapie, mais sans les effets secondaires associés aux rayonnements ionisants. Les cellules saines, moins sensibles au stress oxydatif induit par le plasma, ne sont pas affectées dans ces conditions de traitement.

Des expériences menées sur le côlon avec le même dispositif FE DBD ont confirmé l'effet antitumoral des plasmas froids observé d'abord sur des tumeurs cérébrales. Mais les résultats les plus spectaculaires ont été obtenus avec le Plasma Gun sur des tumeurs orthotopiques de cancers du côlon et du pancréas. (Des tumeurs sont dites " orthotopiques " quand elles sont spécifiques à l'organe sur lequel elles sont greffées.) Les expériences impliquant des tumeurs orthotopiques sont beaucoup plus représentatives de situations cliniques et a priori plus prédictives de la réponse attendue chez l'homme. Bien que les tumeurs soient traitées uniquement sur leur surface accessible, il est important de noter que les analyses ont montré que la mort cellulaire était induite dans l'ensemble du volume tumoral.

Dans les expériences sur le cancer du côlon traité par Plasma Gun, le suivi comparé du groupe de souris contrôle et du groupe de souris traitées in situ (une seule application du Plasma Gun pendant $10 \mathrm{mn}$, sept jours

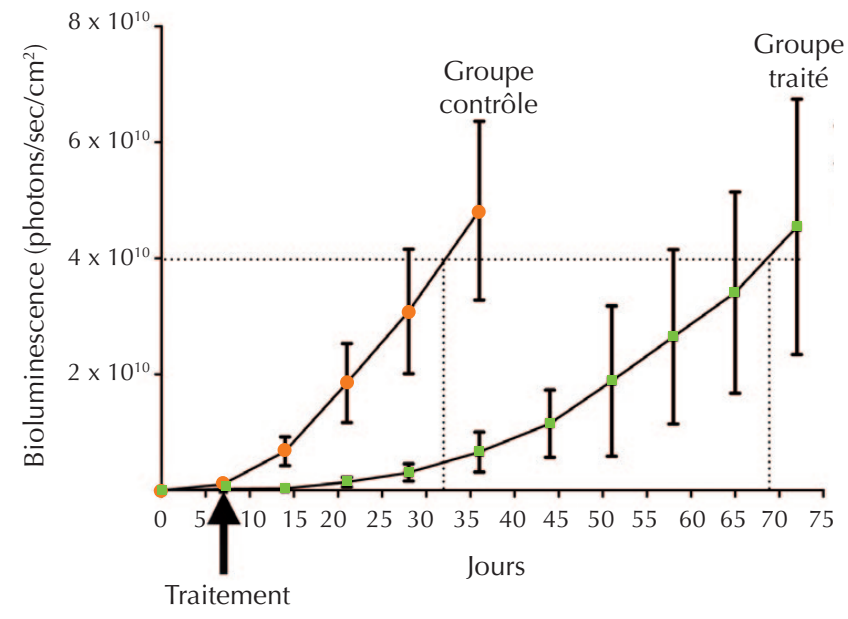

3. Carcinome du côlon. Suivi de la croissance tumorale par imagerie de bioluminescence de tumeurs greffées sur des souris "nude". Comparaison entre un groupe contrôle et un groupe traité au jour 7 (flèche sur la figure) par Plasma Gun à l'hélium $\left(200 \mathrm{~cm}^{3} / \mathrm{s}\right.$ pendant 10 minutes). On estime le gain d'espérance de vie à 115\% pour le groupe traité par Plasma Gun (la durée de survie est définie par l'instant du franchissement d'un seuil de bioluminescence - ici $4.10^{10}$ photons $/ \mathrm{s} / \mathrm{cm}^{2}$ - associé à un volume tumoral (ritique).
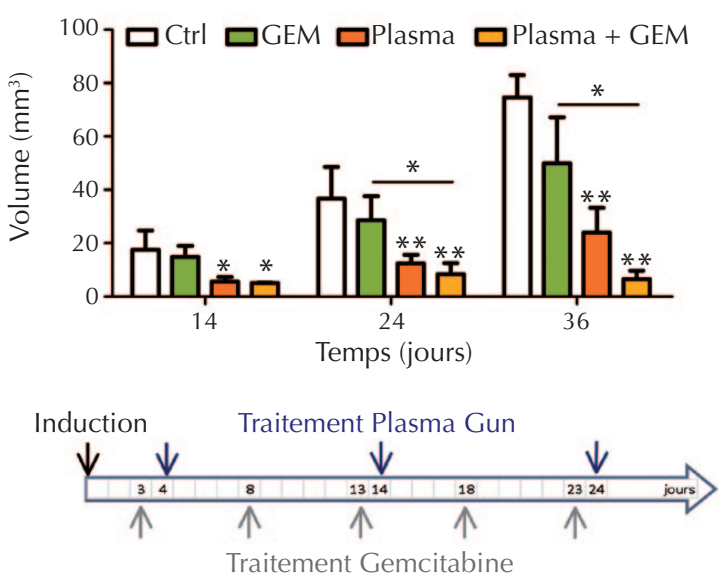

4. Adénocarcinome du pancréas. Comparaison de l'évolution du volume tumoral aux jours 14, 24 et 36 après injection, entre un groupe contrôle (Ctrl), un groupe traité à la gemcitabine (GEM), un groupe traité par Plasma Gun (Plasma) et un groupe ayant reçu un traitement combiné (Plasma + GEM). La séquence des traitements est indiquée sur le diagramme du bas. Une étoile (resp. deux étoiles) sur une barre d'histogramme (ou une ligne horizontale) indique une probabilité de 95\% (resp. 99\%) que la différence avec le groupe de contrôle (ou entre les groupes joints par la ligne) soit significative. 
après le greffage de la tumeur) a montré une très nette réduction de la croissance tumorale chez les souris traitées (fig. 3). Le traitement plasma a entraîné une augmentation de $115 \%$ de leur espérance de vie. Au-delà de la réduction tumorale, ces expériences ont aussi permis de mettre en évidence une très nette réduction, voire une absence dans de nombreux cas, de la prolifération de métastases (voir la photo de l'encadré 2). Les mécanismes à l'origine de ce phénomène ne sont pas encore connus, mais suscitent le plus grand intérêt et feront l'objet d'études à venir.

Le cancer du pancréas est le cancer gastrointestinal ayant le plus mauvais pronostic chez l'homme, avec un taux de survie à 5 ans de l'ordre de 5\%. Afin d'évaluer l'efficacité du traitement par PFA, un modèle basé sur le développement de tumeurs de carcinome pancréatique a été développé en collaboration entre le CERB $^{(a)}$ et le CIPA-TAAM. Des expériences ont été menées sur quatre groupes de souris "nude" porteuses de greffes de tumeurs, suivies par imagerie de bioluminescence in vivo : un groupe contrôle non traité, mais dont les individus ont été opérés comme dans les autres groupes; un groupe traité uniquement par gemcitabine à $200 \mathrm{mg} / \mathrm{kg}$, chimiothérapie de référence pour le cancer du pancréas ; un groupe traité uniquement par Plasma Gun ; enfin un groupe subissant un traitement combiné de gemcitabine et de Plasma Gun. Les résultats (fig. 4) sont extrêmement encourageants. Non seulement ils ont permis pour la première fois de montrer un effet antitumoral des PFA par Plasma Gun sur le pancréas, mais - également pour la première fois - ils ont mis en évidence un effet bénéfique de l'association chimiothérapie/ plasma froid atmosphérique. On peut voir que, 36 jours après l'injection, la réduction du volume tumoral (comparé au groupe contrôle) est plus importante dans le groupe Plasma Gun (68\%) que dans le groupe gemcitabine (30\%). Le résultat le plus frappant est, bien sûr, que le traitement impliquant chimiothérapie et PFA conduit à une réduction encore plus importante de la tumeur, de l'ordre de 90\%.

Ces expériences ouvrent la voie à des traitements combinés sur les zones situées aux alentours immédiats d'une tumeur après son ablation chirurgicale, traitements qui permettraient soit de diminuer les doses de chimiothérapie pour le confort du patient, soit d'améliorer le pronostic vital à moyen terme. Ces travaux vont être poursuivis, afin de déterminer un optimum dans la séquence et les doses des deux traitements. Comme pour le carcinome du côlon, les travaux réalisés sur l'adénocarcinome du pancréas ont aussi permis de montrer dans de nombreux cas une réduction importante de la prolifération des métastases. De nouvelles expériences vont être menées sur ce point particulier, afin de le confirmer et d'essayer de comprendre les mécanismes impliqués.

\section{Vers des applications par voie endoscopique}

L'ensemble des résultats obtenus laisse entrevoir de nombreux champs d'applications thérapeutiques des plasmas. Ceux-ci vont cependant nécessiter des efforts importants de recherche pour une meilleure compréhension des processus impliqués dans les interactions plasma/ cellule et plasma/tissu, pour l'évaluation des effets à long terme, et pour le passage difficile mais indispensable des modèles animaux aux essais cliniques de grande envergure. Parmi toutes les sources de PFA, le Plasma Gun paraît particulièrement adapté à des traitements dans des structures complexes. On pense bien évidemment aux poumons, dont la possibilité de traitement a été démontrée dans le cadre du projet PLASMED, ou à des cibles, notamment tumorales, dans des territoires peu compatibles

\section{Imagerie de bioluminescence}

Le suivi de l'évolution du volume tumoral sur des animaux modèles a longtemps été un problème, dans la mesure où il impliquait leur sacrifice pour réaliser une mesure directe après ablation chirurgicale. Au cours de ces dernières années, les avancées de la bio-imagerie ont permis de pallier ce problème, avec un suivi non invasif et en temps réel grâce à l'Imagerie de BioLuminescence (IBL). Cette technique est maintenant utilisée dans de nombreux domaines tels que la thérapie cellulaire, I'infectiologie, la toxicologie ou la cancérologie. Elle permet la détection in vivo de cellules luminescentes sans apport de lumière extérieure, contrairement à I'imagerie de fluorescence. La bioluminescence naturelle la plus connue est sans doute celle de la luciole, dont la petite lumière émise dans la nuit a capté l'attention de tout un chacun. Cette bioluminescence est due à une réaction chimique, qui fait intervenir deux agents : la luciférine et la luciférase. La luciférase sert de catalyseur à l'activation de la luciférine, qui conduit à la formation d'une molécule oxydée dans un état excité. Sa désexcitation dissociative produit une émission de lumière. Cette lumière émise, même à l'intérieur du corps, peut être facilement détectée par les caméras de grande sensibilité actuellement disponibles.

L'imagerie de bioluminescence est ainsi réalisée à partir de cellules de greffons tumoraux de la lignée dite "luc » exprimant le gène de la luciférase, celui-là même de la luciole. La luciférine est ensuite injectée à l'animal au moment souhaité pour la mesure. L'émission de lumière est alors corrélée à l'activité tumorale. Son enregistrement permet donc de suivre in vivo l'évolution de tumeurs ou la progression de métastases (fig. E2).

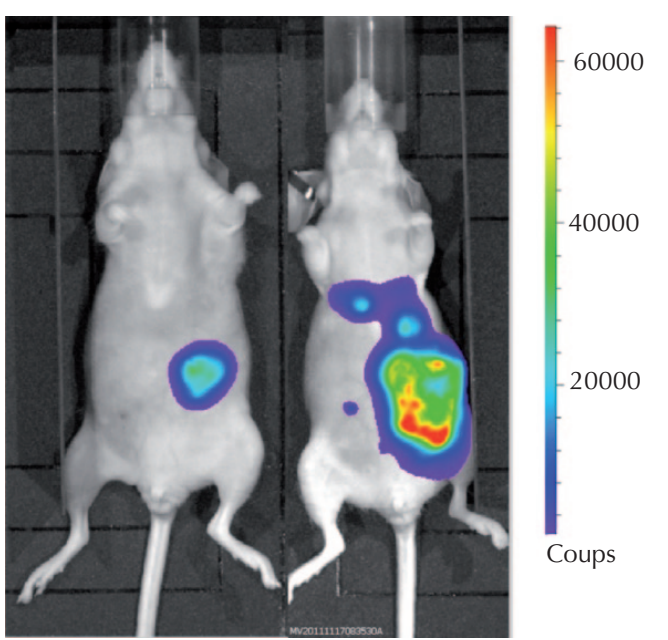

E2. Images de bioluminescence d'une souris traitée par Plasma Gun (à gauche) et d'une souris contrôle (à droite), prises 37 jours après le greffage de la tumeur, dans le cadre des expériences sur le côlon (voir le texte et la figure 3). On peut noter l'absence de métastases dans le cas de la souris traitée par plasma, contrairement à la souris non traitée. 
$\gg>$

avec la chirurgie. Cela implique le développement d'outils endoscopiques intégrant des dispositifs du type Plasma Gun, au côté des instruments traditionnels d'intervention. Le champ de la médecine plasma est à présent ouvert. Il reste aux physiciens et aux ingénieurs un important travail à réaliser pour mettre en œuvre et optimiser des sources de plasma atmosphérique.

Ont participé à ce travail :

Laura Brulé, Sébastien Dozias, Claudine Kieda, Alain Le Pape, Stéphanie Lerondel, Jean-Michel Pouvesle, Delphine Riès, Éric Robert, Vanessa Sarron, Julien Sobilo, Marc Vandamme,

avec le soutien du CNRS, des Universités d'Orléans et de Tours, de l'ANR, de la Région Centre, du Conseil Général du Loiret, du CHR d'Orléans et des entreprises INEL et Germitec.

(a) GREMI : Groupe de Recherches sur l'Énergétique des Milieux Ionisés, UMR 7344 CNRS.

CIPA-TAAM : Centre d'Imagerie du Petit Animal

- Transgenèse et Archivage d'Animaux Modèles, UPS CNRS 44. CBM : Centre de Biophysique Moléculaire UPR CNRS 4301. CERB : Centre de Recherches Biologiques, 18800 Baugy. MPE : Max Planck Institute for Extraterrestrial Physics, Garching, Allemagne.

(b) Espèces réactives de l'oxygène et de l'azote (respectivement ROS, Radical Oxygen Species, et RNS, Radical Nitrogen Species) : espèces chimiques rendues très réactives par la présence d'électrons de valence non appariés. D'origine exogène ou endogène, elles sont capables d'oxyder les protéines, l'ADN ou les lipides des membranes des cellules.

(c) Stress oxydatif : agression des constituants de la cellule, due à des radicaux libres oxydants.

(d) Angiogenèse : processus (normal ou pathologique) de croissance de nouveaux vaisseaux sanguins à partir de vaisseaux préexistants.

(e) Quelques exemples : Plasma Bioscience Research Center, Kwangwoon, Corée du Sud ; Drexel Plasma Medicine Lab, Philadelphie, USA ; Campus PlasmaMed, Greifswald, Allemagne ; Plasma Health Care, MPI, Garching, Allemagne.

(f) Travaux réalisés par le GREMI, associé au CIPATAAM et au CBM, dans le cadre du programme PLASMED.

(g) Une souris "nude" est une souris de laboratoire génétiquement modifiée, au système immunitaire inhibé, permettant le développement de tumeurs humaines greffées pour l'étude des traitements thérapeutiques. 